Check for updates

Cite this: J. Mater. Chem. A, 2020, 8 , 6067

Received 8th November 2019

Accepted 9th March 2020

DOI: 10.1039/c9ta12313j

rsc.li/materials-a

\title{
Metal-organic framework nanosheets for enhanced performance of organic photovoltaic cells $\dagger$
}

\author{
Kezia Sasitharan, ${ }^{a}$ David G. Bossanyi, (D) ${ }^{\mathrm{b}}$ Naoum Vaenas, ${ }^{\mathrm{b}}$ Andrew J. Parnell, (DDb \\ Jenny Clark, ${ }^{b}$ Ahmed Iraqi, ${ }^{a}$ David G. Lidzey (iD b and Jonathan A. Foster iD *a
}

\begin{abstract}
Metal-organic framework nanosheets (MONs) are an emerging class of two-dimensional materials whose diverse and readily tunable structures make them ideal for use in optoelectronic applications. Here, liquid exfoliation is used to synthesize ultrathin zinc-porphyrin based MONs with electronic and optical properties ideally suited for incorporation into a polythiophene-fullerene (P3HT-PCBM) organic solar cell. Remarkably, the addition of MONs to the photoactive layer of a photovoltaic device results in a power conversion efficiency of $5.2 \%$, almost twice that for reference devices without nanosheets with a simultaneous improvement of $J_{\mathrm{sc}}, V_{\mathrm{oc}}$ and FF. Our analysis indicates that the complimentary electronic, optical and structural properties of the MONs allows them to act as a surface to template the crystallization of P3HT leading to a doubling of the absorbance, a tenfold increase in hole mobility and reduced grain size. These results demonstrate the potential of MONs as a tunable class of twodimensional materials for enhancing the performance of a broad range of organic solar cells and other electronic devices.
\end{abstract}

\section{Introduction}

Two-dimensional nanomaterials such as graphene hold enormous potential for use in advanced electronics, energy, separation and composite materials applications. ${ }^{1-3}$ However, the simple chemical composition of many of these materials mean it can be difficult to optimise them for many applications. Metal-organic framework nanosheets (MONs) are an emerging class of two-dimensional nanomaterials composed of organic linkers coordinated to metal-ions or clusters. ${ }^{4-7}$ MONs display the high surface areas and aspect ratios of other twodimensional materials but have a modular structure which readily allows for systematic tuning of their electronic, optical and mechanical properties and introduction of new chemical functionalities. A diverse range of MONs have been formed either bottom-up, via an arrested crystallization in the presence of a templating agent or interface, or "top-down" by mechanical or liquid exfoliation of layered metal-organic frameworks (MOFs). MONs have already shown significant promise for use in a variety of separation, ${ }^{8,9}$ catalysis, ${ }^{10,11}$ sensing, ${ }^{9,12-14}$ and electronics applications. ${ }^{15-19}$

${ }^{a}$ Department of Chemistry, The University of Sheffield, Dainton Building, Brook Hill, S3 7HF, Sheffield, UK. E-mail: jona.foster@sheffield.ac.uk

${ }^{b}$ Department of Physics and Astronomy, The University of Sheffield, Hicks Building, Hounsfield Road, S3 TRH, Sheffield, UK

$\dagger$ Electronic supplementary information (ESI) available. See DOI: 10.1039/c9ta12313j
Organic photovoltaics (OPV) offer a variety of attractive properties over current silicon technology, including highly tuneable structures, low cost production, large area manufacturing capability and the potential to create flexible and semi-transparent devices. ${ }^{20}$ Historically OPV has suffered from low power conversion efficiencies and poor stability but there has been renewed interest thanks to recent innovations such as new materials ${ }^{21,22}$ device architectures ${ }^{23}$ and multicomponent (ternary or quaternary) bulk heterojunction (BHJ) approaches. ${ }^{24-29}$ In the latter case, a wide range of additives have been investigated whose complimentary optical or electronic properties enable them to enhance the range of light absorbed, ${ }^{30-32}$ facilitate more efficient electron or hole transport ${ }^{33}$ or modify the morphology of the devices leading to improvements in performance and stability. ${ }^{34-36}$ Two-dimensional materials are ideal candidates for use as additives thanks to their large surface areas and distinct electronic and optical properties. ${ }^{37-39}$ Transition metal dichalcogenides (TMDCs) in particular have attracted significant interest because of their thicknessdependant opto-electronic properties, ${ }^{\mathbf{4 0}}$ ambipolar charge transport, ${ }^{\mathbf{4 1}}$ chemical stability and direct band-gaps in the single layer form. ${ }^{42}$ However, the difficulties in controlling layer thickness and scalability of these materials have so far limited their use in OPV applications. ${ }^{\mathbf{4 3}}$

MONs possess a variety of characteristics that make them attractive candidates for use in photovoltaics, as outlined in the "MOF electronic roadmap". ${ }^{44}$ Their diverse chemistry and modular structures allow for ready tuning of their optical and 
electronic properties. This means MONs have the potential to perform roles in light absorption, carrier generation and transport within PV devices. MONs have a long range order which could help minimize the traps, dead ends and defects problematic in light-harvesting organic polymers. The nanoscopic dimensions of MONs open up new possibilities for their inclusion within the active layer of thin film devices and the highly anisotropic structure of MONs mean they are predisposed to align parallel to the surface.

Most work using MOFs for photovoltaics has focused on their use in dye-sensitized solar cells where they have been used to reduce structural disorder and increase charge mobility. ${ }^{45-47}$ They have achieved this using either dyes or donor-acceptor molecules as ligands within the MOFs or by incorporating dyes or fullerenes within their pores with power conversion efficiencies reported up to $1.27 \% .^{48,49}$ Epitaxially grown MOF films have also been employed as donor layers in planar lightharvesting devices. ${ }^{50-53}$ MONs have shown promise in a variety of electronic applications, ${ }^{18,54}$ however we are only aware of two reports of their use in solar cells. One recent report showed that tellurophene based MONs can be blended with ZnO-PEIE (polyethylenimine ethoxylate) to form a buffer layer which resulted in a small enhancement in the performance of two OPV devices. ${ }^{55}$ In another recent study, porphyrin based MON films were formed on ITO using a Langmuir-Blodgett approach and impregnated with $\mathrm{C}_{60}$ to form a liquid junction solar cell with quantum efficiency less than $0.1 \%{ }^{56}$ To-date, there have been no-reports of the use of MONs or more generally MOFs within the active layer of bulk-heterojunction OPV devices.

Here, we explore a new approach to enhancing OPV bulk heterojunction devices by blending 2D metal-organic nanosheets along with semi-conducting polymers and fullerene acceptors in the photoactive layer via a simple spin-coating procedure. We hypothesized that zinc-porphyrin based MONs would have good electronic and optical properties for inclusion in OPV devices and used ultrasonic liquid exfoliation to create predominantly monolayer nanosheets. Remarkably, addition of the MONs to a well-studied OPV system was found to result in an almost doubling of the device performance compared to the reference devices without MONs. Detailed studies were therefore undertaken in order to understand the role of the MONs on device performance, charge mobility, absorbance, thin film morphology and crystallinity. On the basis of these observations we were able to put forward a model to explain the improvements in performance seen. We anticipate that the diverse and tunable properties of MONs will enable them to play an important role in enhancing the performance of a wide range of other OPV and electronic devices.

\section{Results and discussion}

\subsection{Synthesis and characterisation of the zinc porphyrin MONs}

The layered MOF $\mathrm{Zn}_{2}$ (ZnTCPP), where TCPP is tetrakis(4carboxyphenyl)porphyrin, was synthesised solvothermally using a procedure adapted from Choi et al. ${ }^{57}$ (Fig. 1a). TCPP was heated with zinc nitrate in a DMF : ethanol mixture at $80^{\circ} \mathrm{C}$ for
$24 \mathrm{~h}$ to produce a black microcrystalline powder. XRPD and other solid-state analysis (Fig. S1-S3, ESI $\dagger$ ) showed a good match for the previously reported $\mathrm{Zn}_{2}(\mathrm{ZnTCPP})$ and analogous structures in literature..$^{13,58-62}$

The layered MOF was suspended in chlorobenzene ( $5 \mathrm{mg} \mathrm{ml}{ }^{-1}$ ), sonicated in an ultrasonic bath for 1 hour and centrifuged at $1500 \mathrm{rpm}$ for $10 \mathrm{~min}$ to remove larger particles. The resulting transparent suspension showed Tyndall scattering (Fig. S4, ESI $\dagger$ ) consistent with the presence of nanoparticles. Atomic force microscopy (AFM) and Transmission Electron Microscopy (TEM) revealed the formation of ultra-thin nanosheets with lateral dimension of $300 \pm 100 \mathrm{~nm}$ and a uniform thickness of $1 \pm 0.3 \mathrm{~nm}$ corresponding to predominantly monolayer thickness (Fig. 1a, S5 and S6, ESI $\dagger$ ). Fast Fourier Transform (FFT) of electron diffraction patterns (Fig. 1c) show lattice fringes with spacing of $1.66 \mathrm{~nm}$ consistent with the Zn-Zn distance in the MON structure (Fig. 1c). Electron energy loss spectroscopy (EELS) showed the zero-electron loss peak to be the most intense with an inelastic mean free path of 0.10 , consistent with the observation of a sample of monolayer thickness (Fig. S6c, ESI $\dagger$ ). It is worth noting that the MONs produced by our simple and scalable approach are thinner and have smaller lateral dimensions than those typically obtained from TCPP with Zinc and other metal ions $(\mathrm{Cu}, \mathrm{Co}, \mathrm{Cd})$ including assembly at liquid interfaces, ${ }^{\mathbf{5 9 , 6 0 , 6 2}}$ intercalation of ligands ${ }^{63}$ and surfactant assisted synthesis. ${ }^{13}$

UV-Vis spectra of the $\mathrm{Zn}_{2}$ (ZnTCPP) MONs in suspension show they are strongly absorbing with a characteristic $\pi-\pi^{*}$ soret band at $435 \mathrm{~nm}$ and Q bands at 564 and $601 \mathrm{~nm}$ (Fig. S7, ESI $\dagger$ ). Fig. 2a shows the thin film UV-Vis spectra on quartz substrates in which the band at $440 \mathrm{~nm}$ is assigned as the $\pi-\pi^{*}$ peak, slightly red shifted compared to the MON in suspension, whilst Q bands are seen at 563 and $605 \mathrm{~nm}$ respectively. ${ }^{64}$ The electrochemical properties of the MONs were studied in acetonitrile using cyclic voltammetry (CV) and the key results are shown in Fig. 2d. The HOMO-LUMO energy level calculations derived from the onset of oxidation and reduction are shown in Fig. S8, ESI. $\dagger$ The nanosheets show an electrochemical band gap of $1.8 \mathrm{eV}$ making it a good material for light harvesting applications. Previous literature reports have shown that a minimum energy offset of $0.3 \mathrm{eV}$ between the LUMO of the donor and acceptor is necessary to facilitate efficient exciton dissociation. ${ }^{65}$ The LUMO level of the MONs was found to be $-3.5 \mathrm{eV}$ which makes it ideally positioned between that of the fullerene electron acceptor [6,6]-phenyl-C61-butyric acid methyl ester (PCBM) at $-3.80 \mathrm{eV}$ and electron donating organic polymer poly(3-hexylthiophene-2,5diyl) (P3HT) at $-3.00 \mathrm{eV} \cdot{ }^{66}$ These finding lead us to evaluate $\mathrm{P} 3 \mathrm{HT}-\mathrm{Zn}_{2}(\mathrm{ZnTCPP})-\mathrm{PC}_{60} \mathrm{BM}$ ternary blend organic photovoltaics where all three of the materials in the active layer display an energy offset in the excess of the $0.3 \mathrm{eV}$ limit. Although the high band-gap of P3HT means that the performance of these systems is ultimately limited, as a well-studied $\mathrm{BHJ}$ system it provides an ideal test-bed to investigate our novel materials and rationally understand any changes in the observed performance.

Solution and thin-film photoluminescence (PL) studies were undertaken in order to understand the energy transfer 
a.

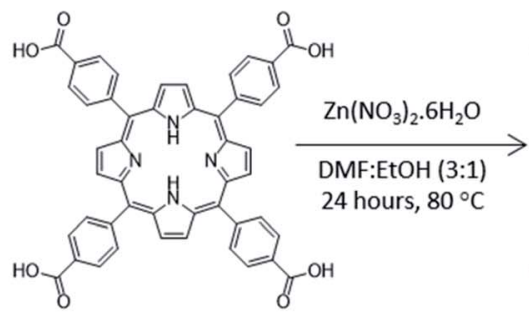

c.

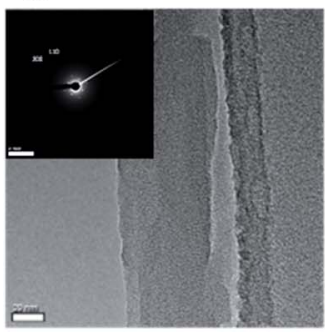

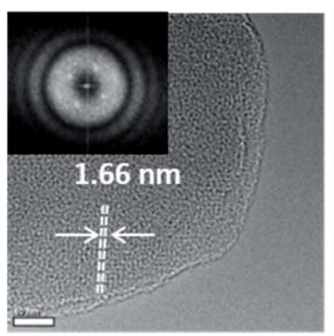

$1.66 \mathrm{~nm}$
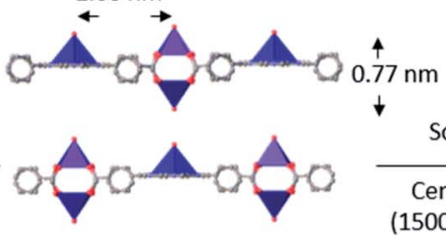

$\downarrow$

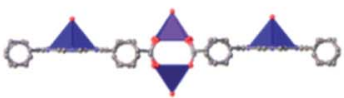

d.

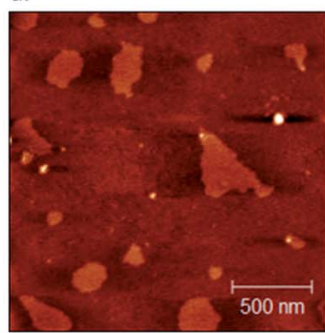

Sonication

Centrifugation (1500 rpm, $10 \mathrm{~min}$

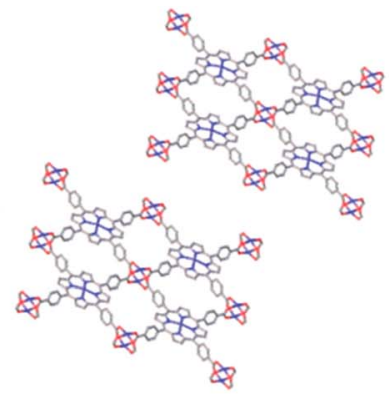

e.
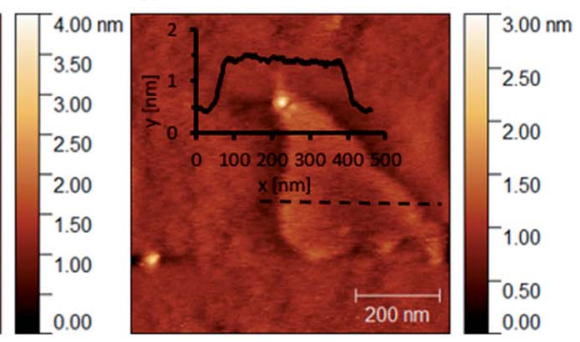

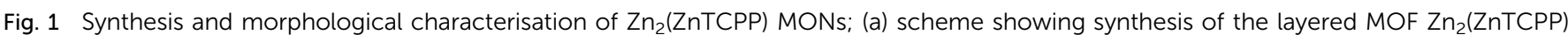
followed by liquid exfoliation into MONs; (b) transmission electron micrograph of a single nanosheet on the TEM grid; (b-inset): single-area electron diffraction pattern of the nanosheets; (c) HR-TEM image of a nanosheet showing lattice fringe distance $=1.66 \mathrm{~nm}$ (inset: the corresponding FFT pattern); (d) AFM topographical image of the MONs as observed under a $1.5 \mu \mathrm{m}$ area scan; (e) AFM image of a single MON with the corresponding height profile as inset $(0.77 \mathrm{~nm}$ thickness; $300 \mathrm{~nm}$ lateral dimension).

characteristics between the MONs and the chosen donoracceptor components. The MONs show a broad PL emission between 500-800 $\mathrm{nm}$ which was quenched upon gradual addition of PCBM (Fig. S10, ESI $†$ ). In the solution state PL, addition of MONs to P3HT resulted in 70\% quenching of the P3HT signal and a $30 \%$ increase in the PL signal of the MONs (Fig. S11, ESI†). This observation is consistent with previous literature studies in ternary blend solar cells. ${ }^{67,68}$ The PL intensity of pristine-P3HT films when compared with that of P3HT-MONs blend films was found to be quenched $(69.6 \%$ quenching a.

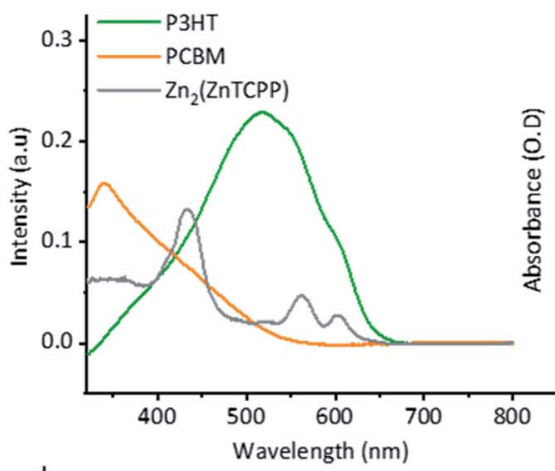

d.

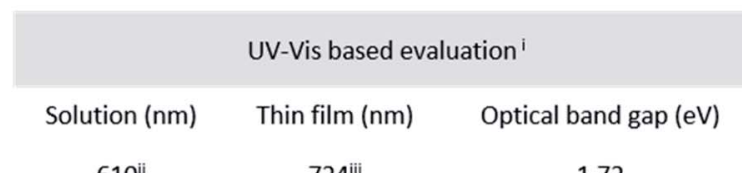

$610^{\mathrm{ii}}$

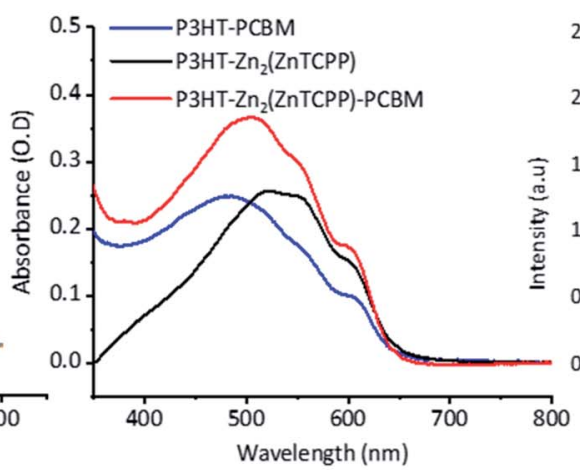

\begin{tabular}{ccc} 
& \multicolumn{2}{c}{ Cyclic voltammetry based evaluation } \\
HOMO $(\mathrm{eV})$ & LUMO $(\mathrm{eV})$ & Electrochemical band gap $(\mathrm{eV})$ \\
-5.3 & -3.5 & 1.80
\end{tabular}

Fig. 2 Optoelectronic characterizations: (a) thin film absorption spectra of P3HT and PCBM spin-coated from chlorobenzene at 2000 rpma60 seconds and $\mathrm{Zn}_{2}(\mathrm{ZnTCPP})$ MONs dropcast $\left(5 \mathrm{mg} \mathrm{ml}^{-1}\right)$ and dried (b) comparison of absorption spectra of the polymer-fullerene, polymernanosheets and polymer-nanosheets-fullerene blends all spin-coated to achieve a layer thickness of 150 nm; (c) photoluminescence spectra recorded from spin coated thin films $(\sim 150 \mathrm{~nm})$ of P3HT, P3HT-Zn ${ }_{2}(Z n T C P P)$ and P3HT-Zn $2(Z n T C P P)-P C B M$ blends, excited at 500 nm. Refer to Fig. S13 (ESI $\dagger$ ) for spin coating parameters used; (d) table showing key results from the band gap evaluation of $Z n_{2}(Z n T C P P) ~ M O N s$ (i) $\lambda_{\text {onset }}$ values obtained by extrapolating the charge transfer peak to the $x$-axis (shown in Fig. S9, ESI†); (ii) the charge transfer UV-Vis peak as observed in Fig. S7; $\uparrow$ (iii) a MON suspension ( $5 \mathrm{mg} \mathrm{ml}^{-1}$ in ethanol) was dropcast onto a quartz substrate, followed by solvent evaporation prior to recording the UV-Vis spectra. 
efficiency, obtained by integrating the area under curve) with the addition of MONs. When $\mathrm{PC}_{60} \mathrm{BM}$ is introduced into the blend, complete quenching of the PL signal is observed (Fig. 2c). Fig. S12, ESI $\uparrow$ shows the systematic study of PL quenching of P3HT thin films upon gradual increase in the weight ratio of incorporated MONs which saturates at a ratio of $1: 0.5$ P3HT: $\mathrm{Zn}_{2}(\mathrm{ZnTCPP})$.

\subsection{Device fabrication, optimisation and performance}

The blends were deposited by spin coating on ITO coated glass substrates containing a 30-40 nm thick layer of PEDOT:PSS. The spin coating parameters were adjusted in order to obtain a 150-200 nm thick photoactive layer. Finally, a $5 \mathrm{~nm}$ thick BCP (2,9-dimethyl-4,7-diphenyl-1,10-phenanthroline, commonly known as bathocuproine) buffer layer and a $100 \mathrm{~nm}$ thick $\mathrm{Ag}$ electrode was thermally evaporated on top and the devices were encapsulated with glass covers. The current density-voltage curves under illumination with a solar simulator (AM 1.5, 1SUN) for the blends P3HT:Zn ${ }_{2}$ (ZnTCPP):PCBM and control P3HTPCBM are shown in Fig. 3b for the optimised device. Remarkably, the power conversion efficiency (PCE) of the best performing optimised devices with nanosheets was $5.2 \%$; the highest value reported in the literature for this type of device (Fig. 3b) and twice that reported for the best performing device without nanosheets $(2.67 \%)$. The best performing optimised device with the nanosheets exhibited a short circuit current density $\left(J_{\mathrm{sc}}\right)$ of $10.8 \mathrm{~mA} \mathrm{~cm}{ }^{-2}$, open circuit voltage $\left(V_{\mathrm{oc}}\right)$ of $0.69 \mathrm{~V}$ and fill factor (FF) of $69 \%$ corresponding to a PCE of $5.2 \%$. These results are also remarkably reproducible with a low standard deviation of $0.12 \%$ PCE across 35 devices developed. The laser
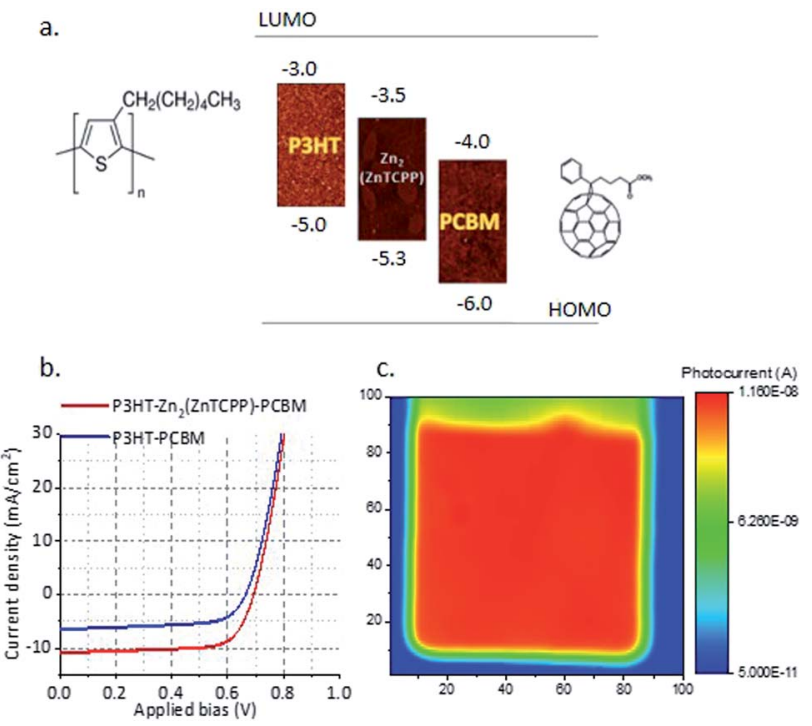

Fig. 3 Device performance metrics; (a) a representation of the HOMO-LUMO level alignment of the components of the ternary device; (b) $J-V$ curve of the best performing devices with and without the MONs; (c) high resolution LBIC mapping of the P3HT-MONPCBM device showing a uniform distribution of current over the entire mapped region, each division on the axes corresponds to the step size $=25$ microns beam induced current (LBIC) mapping of the MON based devices shows uniform current distribution throughout the mapped region (Fig. 3c).

Table S1 (ESI $\dagger$ ) details the iterative development of the devices, varying the ratio of materials and annealing protocols. It is worth noting that as the concentration of MONs in the blend is increased from $1: 0.25: 1$ to $1: 0.5: 1$ (P3HT:Zn ${ }_{2}(-$ ZnTCPP):PCBM) weight ratio (Table $\mathrm{S} 1 \mathrm{a}-\mathrm{d}$ ), $\dagger$ the $V_{\text {oc }}$ remains unchanged within error, and the $J_{\mathrm{sc}}$ increases by $2.08 \mathrm{~mA} \mathrm{~cm}^{-2}$ accompanied by a $2 \%$ increase in the fill factor which corresponds to higher power conversion efficiency. This increase in PCE with the gradual increase in the amount of MONs indicates that the MONs play a significant role in controlling the device performance. Upon further increase of the MON concentration $(1: 1: 1)$, there is a significant drop in the FF and PCE. Thus $1: 0.5: 1$ weight ratio of $\mathrm{P} 3 \mathrm{HT}: \mathrm{Zn}_{2}(\mathrm{ZnTCPP}):$ PCBM was fixed as the optimum blend concentration for the nanosheet based devices. The reference devices produced under the same conditions without MONs were consistently lower across all metrics with the best performing optimised device without nanosheets exhibited a $J_{\mathrm{sc}}$ of $7.09 \mathrm{~mA} \mathrm{~cm}{ }^{-2}, V_{\mathrm{oc}}$ of $0.66 \mathrm{~V}$ and $\mathrm{FF}$ of $57.44 \%$ corresponding to a power conversion efficiency of $2.67 \%$. A comparison with data reported in the literature for P3HT-PCBM based device having active layer-BCP-Ag configuration show a broad spread of values across the 38 device results statistically analysed in this work (Table S2, ESI $\dagger$ ). In addition to remarkably high PCE's, the $J_{\mathrm{sc}}$ values of our devices are notably high in comparison to previous literature reports indicating higher current density with the incorporation of MONs. Importantly, reference devices with the bulk unexfoliated MOF incorporated into the active layer were not found to function, presumably because of short circuiting due to the $\mu \mathrm{m}$ sized MOFs. This establishes that the nanoscopic dimensions of the monolayer MOF nanosheets play a key role in device performance. A control device was also made by incorporating the TCPP ligand in the P3HT-PCBM blend and the performance was comparable with the P3HT-PCBM devices with a PCE of $1.93 \%\left(J_{\mathrm{sc}}=6.5 \mathrm{~mA} \mathrm{~cm}{ }^{-2}, V_{\mathrm{oc}}=0.54 \mathrm{~V} ; \mathrm{FF}=55 \%\right.$ ) (Fig. S16, ESI $\dagger$ ). Progressive enhancement in the $J_{\text {sc }}$ and PCE and no change in $V_{\text {oc }}$ was observed when both sets of devices were subjected to thermal, solvent and solvent + thermal annealing conditions as shown in Fig. 4 and listed in Table S1, ESI. $\dagger$ Interestingly, the fill factor for the as cast MON devices is significantly improved compared to the reference devices but the gap narrows slightly with the additional annealing processes.

\subsection{Absorption and charge mobility}

External quantum efficiency (EQE) measurements were done to compare the amount of current generation from the devices when irradiated in the wavelength range $(400-700 \mathrm{~nm})$. Comparison of EQE measurements for the MON-based devices with the control devices (Fig. 5a) shows enhanced spectral response around 430-450 $\mathrm{nm}$ corresponding to $\pi-\pi^{*}$ transition absorption of the MONs and another well pronounced enhancement at 550-580 $\mathrm{nm}$ corresponding to the $\mathrm{Q}$ absorption 


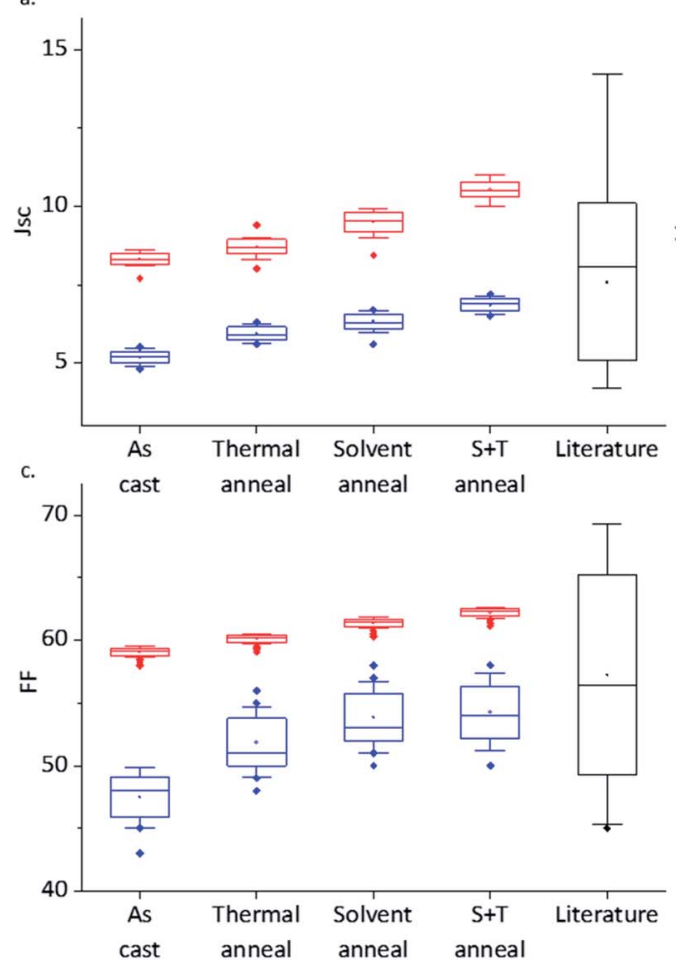

b.

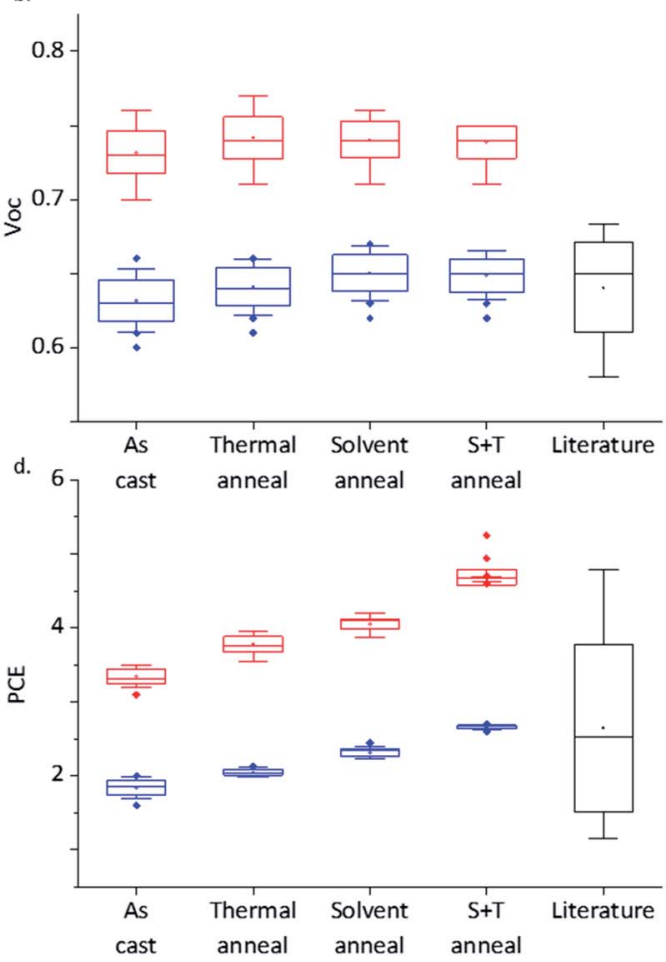

Fig. 4 Statistical analysis of device performance metrics; Comparison of variation of $-J_{\mathrm{sc}}(\mathrm{a}), V_{\mathrm{oc}}$ (b), FF (c) and PCE (d) of the MON based devices (red) with the reference devices (blue) and previous literature reports (black) represented as box plot.

band of the MONs. ${ }^{64} \mathrm{~A}$ higher absorption in the region 600$630 \mathrm{~nm}$ corresponds to an increased percentage of crystalline polymer structures in the film. ${ }^{69} \mathrm{~A}$ decrease in the amorphous P3HT region (Fig. 5b) and the red-shifted $\lambda_{\max }$ of the normalized absorption spectra of the MON incorporated ternary blend (Fig. 5b) supports this analysis.

No obvious new transient-absorption (TA) features were observed when $\mathrm{Zn}_{2}(\mathrm{ZnTCPP})$ was added to the blend. However, the signal for the sample with $\mathrm{Zn}_{2}(\mathrm{ZnTCPP})$ added is roughly twice that of the sample without (Fig. 5c, S17a and b ESI $\dagger$ ). This gives evidence of enhanced light absorption in the blend with the nanosheets, corresponding to higher current density in the solar cell performance. Evaluation of kinetics of the P3HT-PCBM and P3HT-Zn ${ }_{2}$ (ZnTCPP)-PCBM blends show no real change in the excited state dynamics upon addition of $\mathrm{Zn}_{2}$ (ZnTCPP), at least on timescales ranging from $1 \mathrm{ps}$ to $1 \mathrm{~ns}$ (Fig. S18a $\uparrow$ ). However, the kinetics of polaron pairs in P3HT shows that the addition of $\mathrm{Zn}_{2}$ (ZnTCPP) indicates a large increase (more than twofold) in the population of charges in P3HT (Fig. S18b广).

The charge carrier mobility of electrons and holes after dissociation of excitons at the donor/acceptor interface is an important factor in bulk-heterojunction PV performance. Spacecharge limited currents (SCLC) were fabricated and tested in hole only and electron only devices (Table S3 and Fig. S19a$\mathrm{S} 22 \mathrm{~b} \dagger) .{ }^{70}$ In the absence of nanosheets, the electron mobility in P3HT-PCBM is higher by an order of magnitude than the hole mobility. This can lead to unbalanced charge transport and non-geminate recombination. The inclusion of MONs increases the hole mobility of the system by a factor of 10 , improving the balance of hole and electron mobility within the devices. This enables more efficient extraction of charges during transport to the electrodes reducing the opportunity for free charges to encounter each other and recombine at the donor-acceptor interface.

\subsection{Understanding the morphology - GIWAXS and AFM}

Grazing Incidence Wide Angle X-ray Scattering (GI-WAXS) measurements were performed on $\sim 150 \mathrm{~nm}$ thick films with and without nanosheet in order to probe the structure of the thin films used in the devices (Fig. 5d and S23†). Peak at $0.55 \AA$ corresponds to reflection peaks (110) from $\mathrm{Zn}_{2}(\mathrm{ZnTCPP})$ nanosheets ${ }^{57}$ which confirms that the nanosheets do not degrade in the presence of the polymer or during the high temperature anneal but facilitate a well-ordered arrangement of the polymer chains and enhance the crystallinity. The peak corresponding to (100) reflections of $\mathrm{P} 3 \mathrm{HT}$ is observed with an enhanced intensity in the GI-WAXS signal from P3HT- $\mathrm{Zn}_{2}$ (ZnTCPP) film. P3HT appears to have predominantly adopted a face on orientation with respect to the substrate with an intense lamellar stacking peak in plane (100) along $q_{x}$. The crystallite size of P3HT was calculated to be $13.59 \mathrm{~nm}$ from the 100 and 110 peaks using Scherrer analysis (Table S4, ESI $\dagger$ ). These observations indicate a higher fraction of crystalline P3HT upon the inclusion of $\mathrm{Zn}_{2}$ (ZnTCPP), consistent with the observed changes in absorption spectra. 
a.

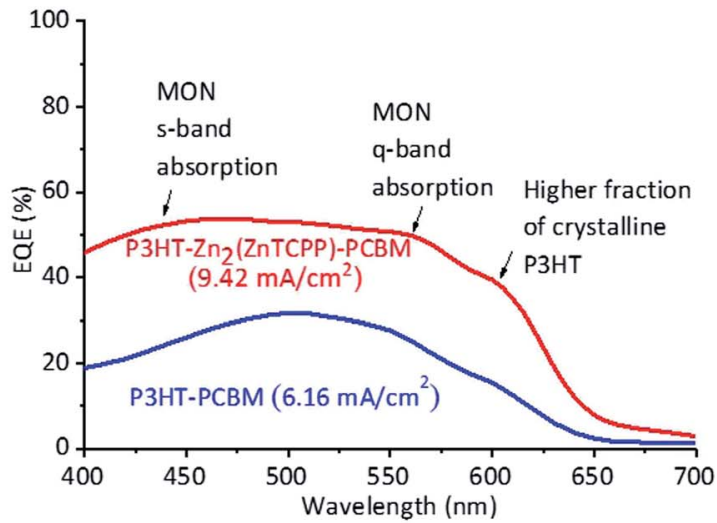

b.

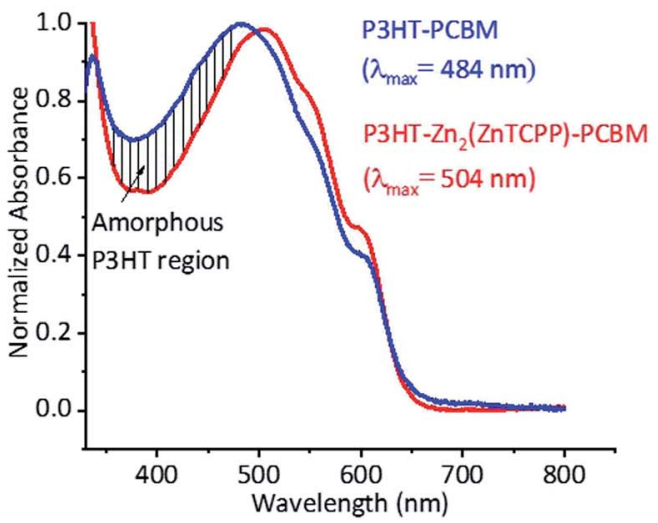

c.

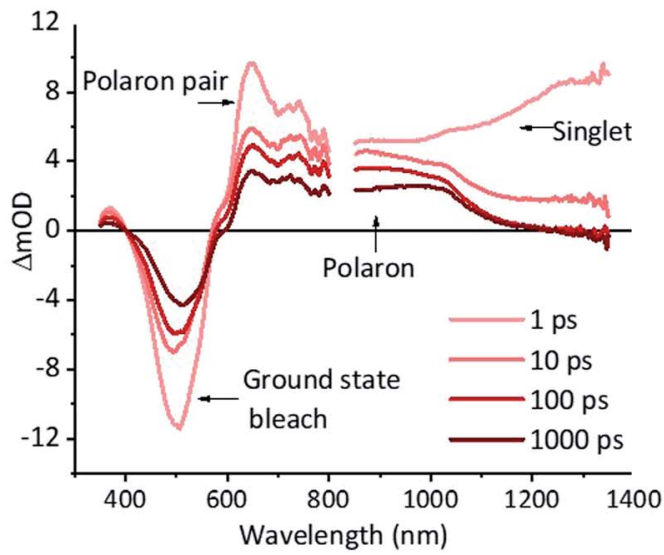

d.

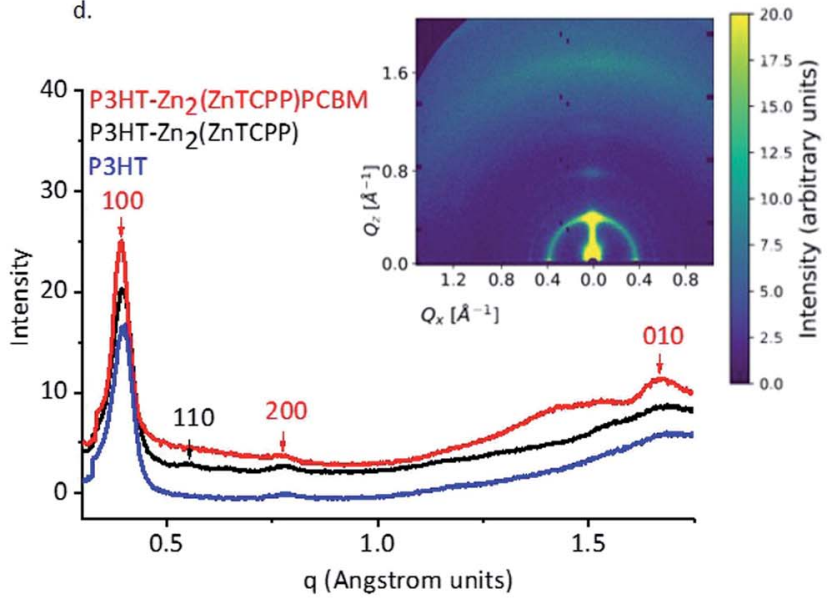

Fig. 5 EQE, absorption and GI-WAXS results: (a) EQE spectra of the MON based devices and the reference devices (with the integrated $J_{\text {sc }}$ values); (b) comparison of the normalized absorption spectra of the reference active layer and the MON based ternary blend-showing a decrease in the amount of amorphous P3HT upon incorporation of MONs; (c) transient absorption spectra of from P3HT-Zn $n_{2}(\mathrm{ZnTCPP})-\mathrm{PCBM}$ blend; (d) the 1D cross-sectional analysis of Gl-WAXS signals from pristine P3HT and P3HT- $\mathrm{Zn}_{2}(\mathrm{ZnTCPP})$ blend film overlaid with the signal from P3HT$Z n_{2}(Z n T C P P)-P C B M$ active layer blend processed according to the device optimisation procedure; (inset: $2 \mathrm{D}$ detector image of P3HT$\left.\mathrm{Zn}_{2}(\mathrm{ZnTCPP})\right)$

AFM imaging of $\mathrm{P} 3 \mathrm{HT}-\mathrm{Zn}_{2}$ (ZnTCPP)-PCBM spin coated blends shows the MONs to be homogeneously distributed throughout the matrix of P3HT-PCBM. Demixing of P3HT and PCBM during thin film formation leads to separate regions of P3HT rich and PCBM rich nanoscale domains. ${ }^{71}$ A spatial Fourier transform was used to extract the phase separation length scales (grain size) from the AFM images (Fig. S25 and S26, ESI $\dagger$ ). In the reference P3HT-PCBM active layer these are in the range of 10-15 $\mathrm{nm}$, while in the presence of MONs the length scale is $6-10 \mathrm{~nm}$. In the presence of MONs, the grain sizes are smaller and more well defined which we speculate favours charge carrier transport. It has been previously shown that the overgrowth of PCBM aggregates (in some literature reports micron sized PCBM crystallites have been observed) correlate with degraded power conversion efficiency and is a reason for detrimental performance in fullerene based systems. ${ }^{72}$ The presence of an additional 2D component which interacts closely with both P3HT and PCBM appears to inhibit their de-mixing and act as a barrier to prevent the formation of oversized PCBM aggregates.

\section{Discussion of the role of MONs within the devices}

The zinc-porphyrin MONs possess a number of features that make them ideally suited for incorporation as third component into the active layer of OPVs. The nanoscopic dimensions of the MONs, predominantly monolayer in thickness and $\sim 300 \mathrm{~nm}$ wide, allows for their incorporation at high concentrations ( $20 \mathrm{wt} \%$ ) within the $\sim 150 \mathrm{~nm}$ thick active layer of the devices. It is worth emphasising that devices based on the bulk MOF did not function and it is only because the predominantly monolayer nanosheets have thicknesses less than the exciton diffusion length that they are able to be incorporated into BHJ type architecture. The porphyrin units have an absorption maximum between that of PCBM and P3HT which means the ternary blends are able to capture a larger fraction of the incident solar spectrum, although this remains dominated by the more strongly absorbing P3HT. The LUMO of the MONs at $-3.5 \mathrm{eV}$ means they are ideally positioned between those of PCBM $(-4.00 \mathrm{eV})$ and P3HT $(-3.00 \mathrm{eV})$ with a greater than $0.3 \mathrm{eV}$ offset 
potentially allowing the MONs to facilitate an energy cascade. However, the transient absorption spectra do not show any of the new features upon inclusion of the MONs that might be expected if the nanosheets are involved in the charge/energy transfer process. This indicates that either charge transfer to the MONs does not happen, or that it occurs faster than the limit of the experimental set-up. Nevertheless, there is typically a trade-off between improvements in $J_{\mathrm{sc}} / \mathrm{FF}$ and $V_{\mathrm{oc}}$ in ternary devices where additives improve device morphology, but the misaligned HOMO-LUMO levels between the corresponding levels of the donor and acceptor materials leads to reduced exciton dissociation and charge transfer rates. The very strong device performance and in particular the fact that the $V_{\mathrm{oc}}$ is close to the theoretical limit for this system indicates that intermediate HOMO-LUMO level of the MONs prevents geminate recombination losses which would otherwise limit device performance.

The addition of nanosheets also impacts significantly on the morphology of the device. Absorbance, EQE, GI-WAXS and ellipsometry studies indicate that incorporation of the nanosheets results in a larger fraction of crystalline P3HT regions whilst smaller P3HT-PCBM grain sizes are seen by AFM. These observations could also account for the increased hole mobility observed in charge mobility studies and the increase in the population of charges in $\mathrm{P} 3 \mathrm{HT}$ observed in transient absorption studies in the presence of MONs. The improved matching of the hole and electron mobilities and smaller grain sizes would reduce the chances for charge carrier recombination during transport to the electrodes. ${ }^{70}$ These observations indicate that the two-dimensional MONs act as a surface to template the formation of a larger fraction of crystalline P3HT domains and barriers to inhibit the overgrowth of PCBM aggregates leading to smaller grain sizes (Fig. 6). Thus the role of the MONs is to enhance the morphology of the active layer without hindering

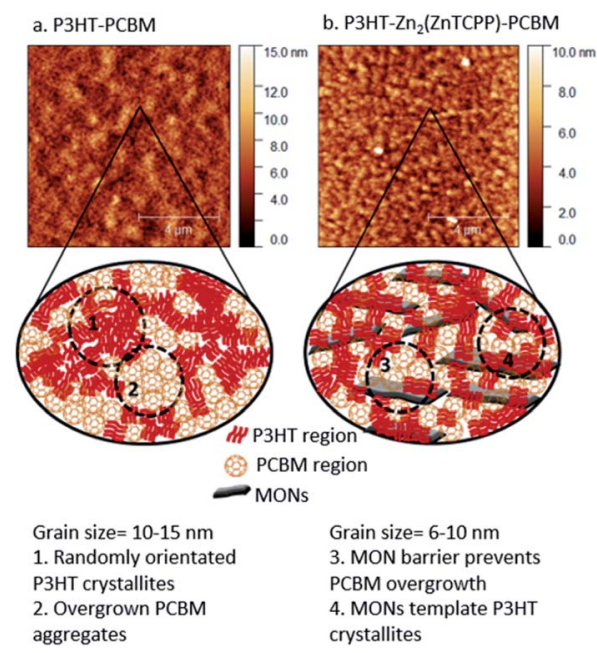

Fig. 6 AFM images showing optimised devices following solvent and thermal annealing with (right) and without (left) MONs and corresponding cartoons highlighting the role of the nanosheets in creating smaller and more crystalline P3HT domains and inhibiting the overgrowth of PCBM aggregates. the formation, dissociation and transport of the charge carriers through the charge percolation pathways.

\section{Conclusions}

Here we have demonstrated the first example of a MON based bulk-heterojunction solar cell by incorporating monolayer $\mathrm{Zn}_{2}$ (ZnTCPP) MONs in the active layer blend of P3HT:PCBM. The MONs' intermediate energy levels with respect to the donor and acceptor system means that they do not create traps for charges, as observed with the introduction of nanomaterials in other ternary systems. The efficiency of the best performing devices with a $20 \%$ loading of MONs was $5.2 \%$, almost double that of the reference devices without nanosheets $(2.67 \%)$ and the highest performing devices of its class. Incorporation of the MONs leads to a significant improvement of the open circuit voltage, current density and fill factor of the solar cells. Absorption, EQE, GI-WAXS, ellipsometry and AFM studies show a tenfold increase in charge mobility, doubling of the absorbance, improved crystallinity of P3HT and reduced P3HT:PCBM domain sizes. We suggest that these observations are best explained by the MONs acting as a template to the formation of P3HT crystallites and as a barrier to prevent the over-growth of PCBM. These results demonstrate the potential of MONs as a new class of two-dimensional nanomaterials for use in optoelectronic devices.

The ease with which the electronic and optical properties of MONs can be tuned mean that the band-gap of these materials can potentially be optimized to blend with any polymer system of choice, potentially with non-fullerene acceptor polymers such as ITIC, IHIC, Y6, PM6 etc. to result in higher performing fullerene-free OPVs. A range of other semi-crystalline OPV devices where MONs can be introduced as ternary blends to enhance crystallinity, improve blending and reducing domain size are immediate candidates for further investigation. However, as a diverse class of readily tunable two-dimensional nanomaterials, MONs have significant potential to play a wide range of roles within OPV and other electronic devices such as OLEDs in order to enhance their performance. For example, MONs may play a role as electron donors or acceptors allowing the creation of binary $\mathrm{BHJ}$ systems, near-IR absorbing ligands can be introduced to allow a wider range of the solar spectrum to be captured and semi-conducting MONs could be used to enhance charge mobility. The low cost, ease of fabrication and the potential to create flexible devices therefore establishes MONs as an important new class of two-dimensional nanomaterials for thin film optoelectronic applications.

\section{Author contributions}

KS carried out the synthesis and characterisation of MONs, device fabrication, testing and related analysis and drafted the manuscript. DGB analyzed the transient absorption data. NV carried out the L-BIC measurement. AJP carried out the GIWAXS studies. JC, AJP, AI and DGL supported experimental design and data analysis and aided in drafting of the 
manuscript. JAF designed and coordinated the project and helped in drafting the manuscript.

\section{Conflicts of interest}

DGL is a co-founder and Chairman of the materials science company Ossila Ltd, that retails materials and equipment used in organic optoelectronic device research and development.

\section{Acknowledgements}

KS thanks the University of Sheffield for a Faculty of Science Scholarship. DGB thanks the EPSRC Centre for Doctoral Training in New and Sustainable Photovoltaics (EP/L01551X/1) for studentship support. DGL thanks the UK EPSRC for partly funding this research via grants EP/M025020/1 'High resolution mapping of performance and degradation mechanisms in printable photovoltaic devices' and EP/J017361/1 'Supersolar Solar Energy Hub. The authors would like to thank Daniel Polak, Alex Auty and Dimitri Chekulaev for assistance with TA measurements and EPSRC for the Capital Equipment award that funded the Lord Porter Laser Facility at the University of Sheffield (EP/L022613/1, EP/R042802/1). The authors would also like to thank Ian Ross for TEM measurements and Emma Spooner, Dr Onkar S. Game, Rachel Kilbride, Joel A. Smith, Dr Michael-Wong Stringer and Dr Benjamin Freestone for useful discussions.

\section{Notes and references}

1 A. K. Geim and K. S. Novoselov, Nat. Mater., 2007, 6, 183-191.

2 K. Leng, I. Abdelwahab, I. Verzhbitskiy, M. Telychko, L. Chu, W. Fu, X. Chi, N. Guo, Z. Chen, Z. Chen, C. Zhang, Q. H. Xu, J. Lu, M. Chhowalla, G. Eda and K. P. Loh, Nat. Mater., 2018, 17, 908-914.

3 I. V. Vlassiouk, Y. Stehle, P. R. Pudasaini, R. R. Unocic, P. D. Rack, A. P. Baddorf, I. N. Ivanov, N. V. Lavrik, F. List, N. Gupta, K. V. Bets, B. I. Yakobson and S. N. Smirnov, Nat. Mater., 2018, 17, 318-322.

4 S. Kim, H. Wang and Y. M. Lee, Angew. Chem., Int. Ed., 2019, 58, 17512-17527.

5 D. J. Ashworth and J. A. Foster, J. Mater. Chem. A, 2018, 6, 16292-16307.

6 M. Zhao, Y. Huang, Y. Peng, Z. Huang, Q. Ma and H. Zhang, Chem. Soc. Rev., 2018, 47, 6267-6295.

7 R. Sakamoto, K. Takada, T. Pal, H. Maeda, T. Kambe and H. Nishihara, Chem. Commun., 2017, 53, 5781-5801.

8 Y. Peng, Y. Li, B. Yujie, H. Jin, W. Jiao, X. Liu and W. Yang, Science, 2014, 346, 1356-1359.

9 A. Pustovarenko, M. G. Goesten, S. Sachdeva, M. Shan, Z. Amghouz, Y. Belmabkhout, A. Dikhtiarenko, T. Rodenas, D. Keskin, I. K. Voets, B. M. Weckhuysen, M. Eddaoudi, L. C. P. M. de Smet, E. J. R. Sudhölter, F. Kapteijn, B. Seoane and J. Gascon, Adv. Mater., 2018, 30, 1707234.

10 J. Huang, Y. Li, R. K. Huang, C. T. He, L. Gong, Q. Hu, L. Wang, Y. T. Xu, X. Y. Tian, S. Y. Liu, Z. M. Ye, F. Wang,
D. D. Zhou, W. X. Zhang and J. P. Zhang, Angew. Chem., Int. Ed., 2018, 57, 4632-4636.

11 T. He, B. Ni, S. Zhang, Y. Gong, H. Wang, L. Gu, J. Zhuang, W. Hu and X. Wang, Small, 2018, 14, 1-6.

12 D. J. Ashworth, A. Cooper, M. Trueman, R. W. M. Al-Saedi, S. D. Liam, A. J. Meijer and J. A. Foster, Chem.-Eur. J., 2018, 24, 17986-17996.

13 Y. Wang, M. Zhao, J. Ping, B. Chen, X. Cao, Y. Huang, C. Tan, Q. Ma, S. Wu, Y. Yu, Q. Lu, J. Chen, W. Zhao, Y. Ying and H. Zhang, Adv. Mater., 2016, 28, 4149-4155.

14 M. Zhao, Y. Wang, Q. Ma, Y. Huang, X. Zhang, J. Ping, Z. Zhang, Q. Lu, Y. Yu, H. Xu, Y. Zhao and H. Zhang, Adv. Mater., 2015, 27, 7372-7378.

15 N. Lahiri, N. Lotfizadeh, R. Tsuchikawa, V. V. Deshpande and J. Louie, J. Am. Chem. Soc., 2017, 139, 19-22.

16 A. Abhervé, S. Mañas-Valero, M. Clemente-León and E. Coronado, Chem. Sci., 2015, 6, 4665-4673.

17 X. Sun, K.-H. Wu, R. Sakamoto, T. Kusamoto, H. Maeda and H. Nishihara, Chem. Lett., 2017, 46, 1072-1075.

18 X. Hu, Z. Wang, B. Lin, C. Zhang, L. Cao, T. Wang, J. Zhang, C. Wang and W. Lin, Chem.-Eur. J., 2017, 23, 8390-8394.

19 G. Wu, J. Huang, Y. Zang, J. He and G. Xu, J. Am. Chem. Soc., 2017, 139, 1360-1363.

20 P. Cheng, G. Li, X. Zhan and Y. Yang, Nat. Photonics, 2018, 12, 131-142.

21 J. Zhao, Y. Li, G. Yang, K. Jiang, H. Lin, H. Ade, W. Ma and H. Yan, Nat. Energy, 2016, 1, 15027.

22 S. Zhang, Y. Qin, J. Zhu and J. Hou, Adv. Mater., 2018, 1800868.

23 Y. Wang, X. Ke, Z. Xiao, L. Ding, R. Xia and H. Yip, Science, 2018, 1098, 1094-1098.

24 C.-H. Tsai, Y.-A. Su, P.-C. Lin, C.-C. Shih, H.-C. Wu, W.-C. Chen and C. Chueh, J. Mater. Chem. C, 2018, 6, 6920-6928.

25 V. Sharapov, Q. Wu, A. Neshchadin, D. Zhao, Z. Cai, W. Chen and L. Yu, J. Phys. Chem. C, 2018, 122, 11305-11311.

26 Y. Cho, T. L. Nguyen, H. Oh, K. Y. Ryu and H. Y. Woo, ACS Appl. Mater. Interfaces, 2018, 10, 27757-27763.

27 M. Nam, H. Y. Noh, J. Cho, Y. Park, S. Shin, J. Kim, J. Kim, H. H. Lee, J. W. Shim and D. Ko, Adv. Funct. Mater., 2019, 1900154.

28 M. Nam, M. Cha, H. H. Lee, K. Hur, K. Lee, J. Yoo, I. K. Han, S. J. Kwon and D. Ko, Nat. Commun., 2017, 8, 1-10.

29 K. Feron, M. N. Thameel, M. F. Al-Mudhaffer, X. Zhou, W. J. Belcher, C. J. Fell and P. C. Dastoor, Appl. Phys. Lett., 2017, 110, 133301.

30 N. Gasparini, L. Lucera, M. Salvador, M. Prosa, G. D. Spyropoulos, P. Kubis, H.-J. Egelhaaf, C. J. Brabec and T. Ameri, Energy Environ. Sci., 2017, 10, 885-892.

31 T. Ameri, J. Min, N. Li, F. Machui, D. Baran, M. Forster, K. J. Schottler, D. Dolfen, U. Scherf and C. J. Brabec, Adv. Energy Mater., 2012, 1198-1202.

32 F. Zhang, Q. An and M. Zhang, Adv. Energy Mater., 2018, 8, 1702854.

33 R. A. Street, D. Davies, P. P. Khlyabich, B. Burkhart and B. C. Thompson, J. Am. Chem. Soc., 2013, 1-4.

34 M. Garg and V. Padmanabhan, Sci. Rep., 2016, 1-12. 
35 Y. Zhang and Z. Wei, Nanotechnology, 2015, 26, 204001. 36 B. Aïssa, M. Nedil, J. Kroeger and A. Ali, Nanotechnology.

37 M. M. Stylianakis, D. Konios, C. Petridis, G. Kakavelakis, E. Stratakis and E. Kymakis, 2D Mater., 2017, 4, 042005.

38 W. Yang, Z. Ye, T. Liang, J. Ye and H. Chen, Sol. Energy Mater. Sol. Cells, 2019, 190, 75-82.

39 R. Ahmad, R. Srivastava, S. Yadav, S. Chand and S. Sapra, ACS Appl. Mater. Interfaces, 2017, 9(39), 34111-34121.

40 H. J. Conley, B. Wang, J. I. Ziegler, R. F. Haglund, S. T. Pantelides and K. I. Bolotin, Nano Lett., 2013, 13, 3626-3630.

41 S. Fan, X. tang, D. Zhang, X. Hu, J. Liu, L. Yang and J. Su, Nanoscale, 2019, 11, 15359-15366.

42 M. M. Ugeda, A. J. Bradley, S. F. Shi, F. H. Da Jornada, Y. Zhang, D. Y. Qiu, W. Ruan, S. K. Mo, Z. Hussain, Z. X. Shen, F. Wang, S. G. Louie and M. F. Crommie, Nat. Mater., 2014, 13, 1091-1095.

43 Q. H. Wang, K. Kalantar-Zadeh, A. Kis, J. N. Coleman and M. S. Strano, Nat. Nanotechnol., 2012, 7, 699-712.

44 I. Stassen, N. Burtch, A. Talin, P. Falcaro, M. Allendorf and R. Ameloot, Chem. Soc. Rev., 2017, 46, 3185-3241.

45 W. A. Maza, A. J. Haring, S. R. Ahrenholtz, C. C. Epley, S. Y. Lin and A. J. Morris, Chem. Sci., 2016, 7, 719-727.

46 S. Jin, H.-J. Son, O. K. Farha, G. P. Wiederrecht and J. T. Hupp, J. Am. Chem. Soc., 2013, 135, 955-958.

47 C. Y. Lee, O. K. Farha, B. J. Hong, A. A. Sarjeant, S. T. Nguyen and J. T. Hupp, J. Am. Chem. Soc., 2011, 133, 15858-15861.

48 D. Y. Lee, E. K. Kim, C. Y. Shin, D. V. Shinde, W. Lee, N. K. Shrestha, J. K. Lee and S. H. Han, RSC Adv., 2014, 4, 12037-12042.

49 E. D. Spoerke, L. J. Small, M. E. Foster, J. Wheeler, A. M. Ullman, V. Stavila, M. Rodriguez and M. D. Allendorf, J. Phys. Chem. C, 2017, 121, 4816-4824.

50 G. O. N. Ndjawa, M. R. Tchalala, O. Shekhah, J. I. Khan, A. E. Mansour, J. Czaban-Jóźwiak, L. J. Weselinski, H. A. Ahsaine, A. Amassian and M. Eddaoudi, Materials, 2019, 12(15), 2457.

51 J. Liu, W. Zhou, J. Liu, Y. Fujimori, T. Higashino, H. Imahori, X. Jiang, J. Zhao, T. Sakurai, Y. Hattori, W. Matsuda, S. Seki, S. K. Garlapati, S. Dasgupta, E. Redel, L. Sun and C. Wöll, J. Mater. Chem. A, 2016, 4, 12739-12747.

52 C. W. Kung, T. H. Chang, L. Y. Chou, J. T. Hupp, O. K. Farha and K. C. Ho, Chem. Commun., 2015, 51, 2414-2417.

53 C. Zhang, X. Li, S. Z. Kang, L. Qin, G. Li and J. Mu, Chem. Commun., 2014, 50, 9064-9067.
54 W. Zhao, J. Peng, W. Wang, S. Liu, Q. Zhao and W. Huang, Coord. Chem. Rev., 2018, 377, 44-63.

55 W. Xing, P. Ye, J. Lu, X. Wu, Y. Chen, T. Zhu, A. Peng and H. Huang, J. Power Sources, 2018, 401, 13-19.

56 C. Liu, C. Wang, H. Wang, T. Wang and J. Jiang, Eur. J. Inorg. Chem, 2019, 4815-4819.

57 E.-Y. Choi, C. A. Wray, C. Hu and W. Choe, CrystEngComm, 2009, 11, 553-555.

58 I. Goldberg, Acta Crystallogr., Sect. A: Found. Crystallogr., 2000, 56, s122.

59 R. Makiura and O. Konovalov, Sci. Rep., 2013, 3, 1-8.

60 R. Makiura and O. Konovalov, Dalton Trans., 2013, 42, 15931-15936.

61 G. Xu, T. Yamada, K. Otsubo, S. Sakaida and H. Kitagawa, J. Am. Chem. Soc., 2012, 134, 16524-16527.

62 S. Motoyama, R. Makiura, O. Sakata and H. Kitagawa, J. Am. Chem. Soc., 2011, 133, 5640-5643.

63 Y. Ding, Y. P. Chen, X. Zhang, L. Chen, Z. Dong, H. L. Jiang, H. Xu and H. C. Zhou, J. Am. Chem. Soc., 2017, 139, 91369139.

64 S. C. Jeoung, D. Kim and D. W. Cho, J. Raman Spectrosc., 2000, 31, 319-330.

65 A. Lefrançois, B. Luszczynska, B. Pepin-Donat, C. Lombard, B. Bouthinon, J. M. Verilhac, M. Gromova, J. FaureVincent, S. Pouget, F. Chandezon, S. Sadki and P. Reiss, Sci. Rep., 2015, 5, 1-8.

66 D. Chen, A. Nakahara, D. Wei, D. Nordlund and T. P. Russell, Nano Lett., 2011, 11, 561-567.

67 M. Koppe, H.-J. Egelhaaf, G. Dennler, M. C. Scharber, C. J. Brabec, P. Schilinsky and C. N. Hoth, Adv. Funct. Mater., 2010, 20, 338-346.

68 E. M. J. Johansson, A. Yartsev, H. Rensmo and V. Sundstro, J. Phys. Chem. C, 2009, 113(7), 3014-3020.

69 J. Clark, J. F. Chang, F. C. Spano, R. H. Friend and C. Silva, Appl. Phys. Lett., 2009, 94, 3-6.

70 J. C. Blakesley, F. A. Castro, W. Kylberg, G. F. A. Dibb, C. Arantes, R. Valaski, M. Cremona, J. Soo and J. Kim, Org. Electron., 2014, 15, 1263-1272.

71 B. A. Collins, J. R. Tumbleston and H. Ade, J. Phys. Chem. Lett., 2011, 2, 3135-3145.

72 W. Wang, S. Guo, E. M. Herzig, K. Sarkar, M. Schindler, D. Magerl, M. Philipp and J. Perlich, J. Mater. Chem. A, 2016, 3743-3753. 